\title{
The Effect of Medical Exercise Therapy on Pressure Sensitivity in Patients with Knee Osteoarthritis: A Cohort Pilot Study
}

Håvard Østerås (D) · Fredrik Paulsberg

Received: December 7, 2018 / Published online: March 30, 2019

(C) The Author(s) 2019

\begin{abstract}
Introduction: While continued research into pain and exercise may address the underlying mechanisms, the mechanisms through which exercise may act are still poorly understood. The purpose of this study was to examine the effects of medical exercise therapy on pressure sensitivity in knee osteoarthritis patients, and to assess whether the decreased pain perception after medical exercise therapy can be explained by changes in pressure sensitivity.

Methods: We adopted acohort design in which 16 patients with long term pain were tested before and after 3 months of medical exercise therapy intervention. Pain was detected by visual analogue scale (VAS) and a digital pressure algometer, which also assessed pressure sensitivity. Function (Knee Osteoarthritis Outcome Score, KOOS), anxiety and depression (HAD) and kinesiophobia (Tampa Scale of Kinesiophobia, TSK) were also measured.
\end{abstract}

Enhanced Digital Features To view enhanced digital features for this article go to: https://doi.org/10.6084/ m9.figshare.7873499.

H. Østerås $(\bowtie)$

Norwegian University of Science and Technology,

Trondheim, Norway

e-mail: havard.osteras@ntnu.no

F. Paulsberg

Rosenborg Clinique, Trondheim, Norway
Results: The VAS showed a statistically significant reduction of perceived pain, from 5.19 (SD 2.04 ) to 4.12 (SD 2.09) from pre- to post-test $(p<0.05)$. There was no significant change in pressure algometry on either the painful side or the non-painful side.

Conclusion: As there were no significant correlations between the reduced pain perception and pressure sensitivity in knee osteoarthritis patients, we suggest that local knee pain does not necessarily alter generalized sensitivity. These findings should be further investigated in a randomized trial in the future.

Trial Registration: Clinicaltrials.gov, identifier NCT02905747.

Keywords: Knee osteoarthritis; Pressure algometer; Sensitivity

\section{INTRODUCTION}

Knee osteoarthritis (OA) is characterized by the presence of an ongoing painful discomfort in the knee, and by the lack of correlations between the overall pain intensity and structural finding, ie degree of MRI- or roentgen verified osteoarthritis [1]. The etiology remains unclear, however, it is currently believed that cross-talk between cartilage and subcondral bone is a critical element, among others [2]. Little is known about pain processing characteristics beyond the immediate knee area in the 
affected patients because of the lack of systemic symptoms and the clinical occurrence of their local pain.

Research indicates that exercise therapy is associated with alterations in perception of pain $[3,4]$. In general, investigators have typically found that hypoalgesia (i.e., reduced sensitivity to noxious stimulation) occurs during and following exercise in healthy adults. This exerciseinduced hypoalgesia has been described by elevations in pain thresholds and pain tolerance, as well as reductions in pain ratings during and following exercise [4]. Most of the research in this area has involved aerobic exercise such as cycling and running, and results have indicated that exercise-induced hypoalgesia occurs most consistently during and following higher intensity aerobic exercise (e.g., > 70\% maximal aerobic capacity) $[4,5]$. There have been a number of studies conducted examining whether isometric exercise may alter pain perception. The results have indicated that exerciseinduced hypoalgesia can occur during and following short duration (e.g., 1.5-5 min), lower intensity (e.g., 15-50\% max), and isometric exercise [6-8]. However, there is little knowledge on the long-term effect of exercise therapy on allodynia and pain sensitization.

Recent research, however, suggests that patients with knee OA have demonstrable differences in sensory processing in peripheral regions as well as at the knee [9]. The finding that patients with knee OA display diffusely increased pain sensitivity in these neutral sites provides evidence for a changed paradigm in treating this problem. This implies that some individuals with this condition might benefit from treatments aimed at more central nervous causes of pain (e.g., certain antidepressants, aerobic exercise and cognitive behavioral therapy), in addition to or instead of therapies directed at the knee. In addition, there is currently limited knowledge about if, and possibly how, pain sensitization can be modified over months in long-term pain patients such as OA patients. Therefore, we sought to further assess the characteristics of the underlying pain in patients with knee OA by evaluating pressure pain sensitivity throughout the knee region compared to the pressure pain sensitivity in a "neutral" peripheral site (ankle). The other aim of the study was to evaluate the possible treatment effect of medical exercise therapy to the pain sensitivity in these patients.

\section{METHODS}

A cohort design was used to describe pain changes before and after 3 months of medical exercise therapy. Data collection took place at a physiotherapy clinic in Trondheim, Norway. OA patients who were prescribed for physiotherapy were asked to participate in the study. The subjects were recruited from August 2017 to April 2018, referred from their general practitioners. They signed a written consent and got oral and written information about the trial. It was emphasized that patients participating voluntarily and without influence management could withdraw from the study at any time. Attending physiotherapists had self-care arrangements.

Inclusion criteria were age between 45-85 years, X-ray verified knee OA (Kellgren \& Lawrence grade I-III) [10] and loss of function and withstanding pain over 3 months. Exclusion criteria were physical therapy treatment during the last 3 months, knee injury, bilateral symptoms, inflammatory arthritis, symptoms from the hip joints greater than the knee pain, planned knee surgery in the next 6 months, and lung and heart disorders or other health issues affecting the ability to complete the intervention. The demographics of the 16 included patients are described in Table 1. A larger, multimodal sensory evaluation was planned to follow this smaller study, depending on results obtained.

\section{Assessments}

All assessments were done before and after the 3-month medical exercise therapy intervention. Pain (average pain last week) was assessed by visual analogue scale (VAS), consisting of a $10 \mathrm{~cm}$ line from "no pain" to "worst pain I can imagine" [11]. Three questionnaires before were completed: fear of pain, anxiety and depression, and knee pain and function. Anxiety and 
Table 1 Descriptive statistics of the participants

\begin{tabular}{llc}
\hline Variables & \multicolumn{2}{l}{ Exercise group $(\boldsymbol{n ~ 1 6})$} \\
\cline { 2 - 3 } & Mean & SD \\
\hline Age (years) & 62.3 & 10.2 \\
Height $(\mathrm{cm})$ & 176.9 & 8.1 \\
Mass $(\mathrm{kg})$ & 84.4 & 11.1 \\
Body mass index $\left(\mathrm{kg} / \mathrm{m}^{2}\right)$ & 27.2 & 4.8 \\
Sex: men (\%) & 56 & \\
Duration of symptoms (years) & 6.8 & 4.7 \\
TSK (kinesiophobia) & 38.8 & 7.9 \\
HADS-A & 7.7 & 3.5 \\
HADS-D & 2.6 & 3.2 \\
KOOS & 42.9 & 17.1 \\
\hline
\end{tabular}

$S D$ standard deviation

depression were assessed by HAD [12], a brief questionnaire to identify possible causes of anxiety and depression. It includes an Anxiety subscale (HADS-A) and a Depression subscale (HADS-D), each containing seven items asking how subjects felt during the last week. Every item was scored $0-3$, depending on the severity of the actual symptom, giving a maximum score of 21 for each of the subscales. A sum score for each domain categorized the person into one of three "risk areas"; (1) 0-7 points, indicating little risk for suffering from anxiety and/or depression, (2) 8-10 points, showing some risk for suffering from anxiety and/or depression, (3) 11-21 points, suffering from anxiety and/or depression. A change from a group of higher points to a group of lower points indicated a clinically meaningful change on a group level. TSK [13] is a widely used instrument to assess fear of movement and (re)-injury. The TSK has 17 items, with items $4,8,12$, and 16 being scored reversely. Each item is scored on a 4-point Likert-type scale. Scoring possibilities range from strongly disagree $($ score $=1)$ to strongly agree $($ score $=4)$. Total TSK scores range between 17 and 68 points, with a cutoff at 37 points, which signifies the median= risk for kinesiophobia.
Pain and function were described as a selfreported composite measure; KOOS comprises five different subscales; (a) pain, (b) other symptoms, (c) activities in daily living, (d) function in sport and recreation and (e) knee related quality of life (QOL) [14]. All items have five possible answer options scored from zero (no problems) to four (heavy problems). The scores were transformed to a 0-100 scale, where 100 represents no knee-related problems. The KOOS is a valid and reliable patient-relevant questionnaire for patients with knee injury and knee osteoarthritis [14]. To detect an average difference between individuals and between groups, a minimal perceptible improvement was set to ten points.

A pressure algometer was used to identify the pressure/force eliciting the pressure-pain threshold (SBMedic Electronics, Sweden). It has been shown reliable and valid [15]. The handheld algometer had a one $\mathrm{cm}^{2}$ round application surface, and the maximum tolerable force at the medial knee and ankle was measured right before and a few minutes after the $30 \mathrm{~min}$ intervention. Both legs were tested. Pressure was applied at the medioventral joint line of the knee and at the medial malleolus, first at the non-painful side followed by the painful side. The subjects were lying on the side, while the bottom limb in this position was tested. The test was terminated, and the result recorded, when the subjects indicated the stimuli was perceived as painful. An average of the three recordings comprised the data for analysis in this study. The procedure was performed at pre- and posttest.

The intervention consisted of a medical exercise therapy program developed for several previous trials in knee patients [16, 17], with focus on coordination and muscle function training, along with pain modification training. Improved coordination and muscle function could potentially have positive influence on knee pain and, finally, the progression of OA. The exercise program allowed for individual differences, as far as possible, due to performance and progression. Based on clinical experience, the intervention period was three months, and the subjects performed the exercise program three times per week. Symptoms 
and other clinical findings were the basis for choosing individual starting positions, range of motion, and weight resistance for each exercise [16]. The exercise group was asked to perform three sets of 30 repetitions. The program was a combination of global aerobic exercises using a stationary ergometer cycle and treadmill, and semiglobal and local exercises to modulate pain and increase range of motion using specially designed exercise equipment. This included wall pulleys, and quadriceps and hamstrings muscle strength training apparatus [16]. There was an $80 \%$ threshold for inclusion that involved completion.

\section{Statistics}

Paired $t$ tests were performed for the comparison of means. Pearson's correlations were performed as secondary analysis between pain and pressure algometry variables, TSK- and HADSscores. Levels of significance were set to $p<0.05$. All the data were tested for normality using a Shapiro-Wilk test, and the assumptions were met for using a $t$ test in most variables. Wilcoxon signed rank tests were used if assumption of normality was violated. Results are presented as mean and standard deviation in text, figures and tables, unless otherwise stated.

This study was registered in clinicaltrials.gov, identifier NCT02905747. This study was approved by The Regional Committees for Medical and Health Research Ethics in Norway, identifier 2016/872. All procedures performed in this study were in accordance with The Regional Committees for Medical and Health Research Ethics in Norway and with the 1964 Helsinki Declaration and its later amendments or comparable ethical standards. Informed consent was obtained from all individual participants included in the study.

\section{RESULTS}

The patients completed all visits. There was a statistically significant reduction of perceived pain at VAS from 5.19 (SD 2.04) to 4.12 (SD 2.09) from pre- to post-test in this cohort $(p<0.05)$; see Table 3 . There was no significant change in pressure algometry on either the painful side or the non-painful side (Tables 2, 3 ). At baseline, the subjects scored mean 38.8 (SD 7.9) at TSK, slightly above the risk of kinesiophobia. The subjects scored mean 7.7 (SD 3.5) at HAD, between risk area 1 and 2 (of 3)

Table 2 Change from baseline to end of intervention, self-reported pain and pressure algometry

\begin{tabular}{|c|c|c|c|}
\hline \multirow[t]{2}{*}{ Variables $(n=16)$} & \multicolumn{2}{|c|}{ Change in score from pre- to post-test } & \multirow[t]{2}{*}{$p$ value } \\
\hline & Mean (SD) & $95 \% \mathrm{CI}$ & \\
\hline Pain (VAS: $0=$ no pain, $10=\max$ ) & $-1.06(1.48)^{\mathrm{a}}$ & $(0.27,-1.85)$ & 0.012 \\
\hline HADS-A & $0.94(1.91)$ & $(-0.08,1.96)$ & 0.069 \\
\hline HADS-D ${ }^{b}$ & $0.31(1.08)$ & $(-0.27,0.89)$ & 0.272 \\
\hline TSK & $2.00(5.02)$ & $(-0.67,4.67)$ & 0.132 \\
\hline \multicolumn{4}{|l|}{ Pain (pressure algometer) $(\mathrm{kPa})$} \\
\hline Painful knee & $8.6(211.0)$ & $(-103.7,121.0)$ & 0.87 \\
\hline Malleolus painful side & $10.9(215.4)$ & $(-103.9,125.7)$ & 0.84 \\
\hline Non-painful knee & $12.3(310.1)$ & $(-177.6,152.9)$ & 0.88 \\
\hline Malleolus non-painful side & $50.4(122.6)$ & $(-115.7,15.0)$ & 0.12 \\
\hline
\end{tabular}


Table 3 Mean (SD) of pressure algometry data with comparison of means between anatomical locations and VAS with comparison of mean pre- and post-intervention

\begin{tabular}{lll}
\hline Site $(\boldsymbol{n}=\mathbf{1 6})$ & $\begin{array}{l}\text { Pre-intervention } \\
\text { (baseline) }\end{array}$ & $\begin{array}{l}\text { Post-intervention } \\
(\mathbf{1 2} \text { weeks })\end{array}$ \\
\hline Painful knee & $718.0(332.8)$ & $726.7(402.4)$ \\
$\begin{array}{l}\text { Malleolus } \\
\text { painful side }\end{array}$ & $473.1(257.6)$ & $483.9(221.1)$ \\
$\begin{array}{l}\text { Non-painful } \\
\text { knee }\end{array}$ & $807.4(410.9)$ & $819.7(447.3)^{\mathrm{a}}$ \\
$\begin{array}{l}\text { Malleolus non- } \\
\text { painful side }\end{array}$ & $480.9(266.8)$ & $531.3(289.8)^{\mathrm{b}}$ \\
\begin{tabular}{l} 
VAS, pain \\
\hline
\end{tabular} & $5.19(2.04)$ & $4.12(2.09)^{\mathrm{c}}$ \\
\hline
\end{tabular}

a Painful- vs non-painful knee, $p<0.05$

b Malleolus painful vs non-painful side, $p<0.05$

c Pre- vs post-intervention, $p<0.05$

(Table 1). There were no statistical differences in TSK or HAD from pre- to post-test.

KOOS-activity of daily living and KOOSsport/recreational activity subscores were both significantly higher at post-test, with a mean change in score of 7.3 (SD 6.5, $p<0.05$ ) and 6.1 (SD 7.4, $p<0.05$ ), respectively.

No significant correlations were found between pain and pressure algometry delta values for the different anatomic test sites $(r=-0.040$ to $0.294, p>0.05$, not shown in table), nor between delta pain and delta HADSA $(r=-0.469, p>0.05)$ or HADS-D $(r=0.279$, $p>0.05)$. However, there was found a significant correlation between change in pain and change in TSK scores $(r=-0.511, p=0.043)$ this correlation was not statistically significant when removing the one most extreme outlier $(r=-0.248, p=0.373)$.

\section{DISCUSSION}

There was no statistically significant increase of the sensory threshold or pain tolerance, as detected by pressure algometer, throughout the study. There was a reduction of VAS, which correlated with increase of kinesiophobia.
However, the latter notion should be interpreted with caution, as such correlations on small datasets are highly susceptible to outliers-as demonstrated through removing the one most extreme outlier, resulting in a nonsignificant correlation. As there is a consensus of using exercise therapy in the treatment of $\mathrm{OA}$ patients, this study adds support to a pain modulation mechanism in medical exercise therapy, though it is not correlated to tolerance for mechanical pressure at the knee nor at the ankle.

Increased pain sensitivity outside the areas of clinical pain has been reported for other regional pain syndromes, such as tension-type headache [18], temporomandibular disorder [19], and localized trapezius myalgia [20]. Hyperalgesia represents an appropriate increase in vigilance to prevent potential harm, and could be explained by the activation of cutaneous polymodal nociceptors in the skin sensitive to heat, pressure and sympathetic stimulation [21]. In therapy, it is generally of great importance to investigate both local and central factors that might explain the overall symptoms of the patients. The more widespread and generalized the pain, the less important local findings, for example in the knee, which in turn might have implications for the treatment package and priorities.

The mechanisms of knee OA-related pain are complex [22], particularly in chronic pain conditions where pain experience is believed to be more a result of changes in the nervous system than in tissue structures [1], which somehow reflects a paradigm shift in the understanding of the pathology of pain related to knee OA. Because of the plasticity of the nervous system, pain lowers the threshold level of the nociceptive receptor system [23], making it more sensitive to stimuli during normal movements like walking, which is loading allodynia. These changes occur in the peripheral receptor system located in the knee and in the receptor system in the spinal cord resulting in changes in the nervous system, i.e., peripheral and central sensitization [24]. As the pain perception may be more related to the nervous system than the knee, it is easier to understand why there are low correlations between structural 
degenerative changes of the knee, pain, and functioning [25]. However, this study suggests pain modulation, though not explained by changes in the central perception of pressure sensitivity.

Physical exercise is an important component in the treatment and rehabilitation of many patients with long-term pain, as well as vital to the overall health and well-being of any individual [26]. The predicted effects of exercise in achieving pain modulation, alone, are an indication for clinical use; although the underlying mechanisms are poorly documented, good pain modulating effects are observed in clinic [27]. According to Bergmann et al. [28], a central pain sensitization may cause changes in the diffuse noxious inhibitory control system. This afferent/efferent system operates via the sensory nociceptive nerve system, and a defect in the diffuse noxious inhibitory control system seems to lead to a state of more diffuse hyperalgesia, as seen in many chronic pain conditions such as chronic osteoarthritis [28].

Pain sensitivity has been found to be changed following exercise. A number of investigators have found diminished sensitivity to pain (hypoalgesia) during and following exercise [i.e. 4]. Importantly, laboratory studies have found that acute exercise reduces sensitivity to painful stimuli in healthy individuals, indicating a hypoalgesic response. This phenomenon has been termed exercise-induced analgesia or exercise-induced hypoalgesia [4]. Hypoalgesia is a decreased sensitivity to painful impact [29], and hypoalgesia can probably be activated at both central and spinal levels during physical exercise. One anticipated mechanism explaining hypoalgesia during exercise is blocking nociceptors from liberated opioids [5]. Another potential mechanism is an interaction between central pain modulation and the cardiovascular system [27]. For example, control of pain and blood pressure involve the same neurons in the brainstem and the same neurotransmitters (e.g., monoamines). A third possible mechanism for exercise-induced hypoalgesia is activation of ascending (e.g., activation of muscle afferent Aand C-fibers) and descending (e.g., activity-induced distraction and changed attention) pathways in the spinal cord [27]. The present study, however, was not able to detect any longterm effect of exercise therapy on pain, as measured by pressure algometer.

It is currently unclear what type of exercise, as well as the duration and intensity of the exercise, that is necessary and sufficient to influence tolerance to nociceptive stimuli [27], particularly over long periods (months). There are studies on people without pain showing that endurance training provides greater tolerance for sustained nociception, and that higher intensity provides greater pain tolerance than low intensity endurance training [5]. In patients, it might be of great importance to assess more knowledge in this field, especially since a majority might not be able to perform high-intensity strength and/or endurance training. As medical exercise therapy consists of many repetitions of the exercises-though relative low intensity-one might expect a significant influence over long time, as tested in the present study.

An understanding of how exercise may influence pain perception is necessary to optimize the clinical utility of exercise therapy as a method of pain management. Several experimental studies have investigated the possible effects of acute exercise as a response to induced noxious stimulation. A variety of exercise modalities, as well as a variety of pain induction techniques and measurement procedures, have been included in these trials. Exercise modalities have included aerobic and isometric exercise, as well as dynamic resistance exercises [27]. Isometric exercises involve a static contraction without any change in the joint angle. Dynamic resistance exercises involve muscle contractions that produce joint movement. These different exercise modes have variations across dimensions such as the type, intensity, and duration of the exercise. Also, techniques of how pain has been induced have included thermal, pressure, electrical and other forms of noxious stimulation [30]. There is, however, still a lack of knowledge as to whether the same phenomenon is present in pain patients, though the present study suggests that there is, but not expressed by changes in pressure sensitivity.

Context may be an important factor in clinical pain measurement [31]. There is hardly 
a measure of the tissue trauma that causes pain, i.e., the local tissue damage does not explain the whole pain response. The patient nevertheless rarely receives training in how to scale pain. Thus, it may be a measure of pain intensity, colored by the emotional experience of pain. Pressure algometry is a golden standard in research of pain, but a major limitation will always be the fact that the measure is based on a subjective sensation of pain.

Several research groups have reported a reduction in temporal summation of pain following physical activity, in line with the development of hypoalgesia [i.e. 32]. Temporal summation of pain is commonly used to reflect the amount of the central nervous system-involved nociception. Central nervous systeminvolved nociception is often hypothesized as being sensitive to increases in both acute and chronic pain, suggesting that exercise may provide benefits in both the central and peripheral nervous system pathways important for pain [33].

A Cochrane review [34] concludes that there is high-quality evidence for the use of exercise as a longtime pain modulator for knee OA. Furthermore, they found moderate-quality evidence for the use of exercise to improve function. The Osteoarthritis Research Society International (OARSI), as a non-pharmacologic modality [35], also recommends exercise.

\section{Limitations}

A lack of healthy controls' response to the same tests is a limitation in this study. Another possible limitation is the reduced standardization in the point of pressure of the pressure algometer, both in the knee and the ankle. A lack of significant pain history is also a limitation.

\section{CONCLUSION}

There were no changes in pain sensitivity after three months of medical exercise therapy in knee osteoarthritis patients, though they achieved significant pain. We, therefore, suggest that local knee pain does not alter generalized sensitivity in these patients. To clarify the role of the central sensory processing alteration in the response to exercise treatment and pathogenesis, further studies are needed. Future research should examine the use of medical exercise therapy in a clinical context, trying to answer what changes that might occur in different neural compartments that underlie pain reduction.

\section{ACKNOWLEDGEMENTS}

We would like to thank the experienced physiotherapists Anders Bakken and Lasse Haugerud for their important contributions in testing and treatment of the patients in this study.

Funding. No funding or sponsorship was received for this study or publication of this article. The article processing charges were funded by the authors.

Authorship. Both named authors meet the International Committee of Medical Journal Editors (ICMJE) criteria for authorship for this article, take responsibility for the integrity of the work as a whole, and have given their approval for this version to be published.

Disclosures. Håvard Østerås and Fredrik Paulsberg have nothing to disclose.

Compliance with Ethics Guidelines. This study was approved by The Regional Committees for Medical and Health Research Ethics in Norway, identifier 2016/872. All procedures performed in this study were in accordance with The Regional Committees for Medical and Health Research Ethics in Norway and with the 1964 Helsinki Declaration and its later amendments or comparable ethical standards. Informed consent was obtained from all individual participants included in the study.

Data Availability. The dataset generated and analyzed during the current study is available from the corresponding author on reasonable request. 
Open Access. This article is distributed under the terms of the Creative Commons Attribution-NonCommercial 4.0 International License (http://creativecommons.org/licenses/ by-nc/4.0/), which permits any noncommercial use, distribution, and reproduction in any medium, provided you give appropriate credit to the original author(s) and the source, provide a link to the Creative Commons license, and indicate if changes were made.

\section{REFERENCES}

1. Arendt-Nielsen L. Joint pain: more to it than just structural damage? Pain. 2017;158(Suppl 1):S66-S73.

2. Fusco M, Skaper SD, Coaccioli S, Varrassi G, Paladini A. Degenerative joint diseases and neuroinflammation. Pain Pract. 2017;17(4):522-32.

3. Drury DG, Greenwood K, Stuempfle K, Koltyn K. Changes in pain perception in women during and following an exhaustive incremental cycling exercise. J Sports Sci Med. 2005;4(3):215-22.

4. Koltyn KF, Brellenthin AG, Cook DB, Sehgal N, Hillard C. Mechanisms of exercise-induced hypoalgesia. J Pain. 2014;15(12):1294-304.

5. Naugle KM, Naugle KE, Fillingim RB, Samuels B, Riley BS, Riley JL. Intensity thresholds for aerobic exercise-induced hypoalgesia. Med Sci Sports Exerc. 2014;46(4):817-25.

6. Hoeger Bement MK, Dicapo J, Rasiarmos R, Hunter SK. Dose response of isometric contractions on pain perception in healthy adults. Med Sci Sports Exerc. 2008;40(11):1880-9.

7. Kosek E, Ekholm J. Modulation of pressure pain thresholds during and following isometric contraction. Pain. 1995;61(3):481-6 (Erratum in: Pain 1996;64(3):603).

8. Kosek E, Lundberg L. Segmental and plurisegmental modulation of pressure pain thresholds during static muscle contractions in healthy individuals. Eur J Pain. 2003;7(3):251-8.

9. Bartley EJ, King CD, Sibille KT, et al. Enhanced osteoarthritis: potential sex differences in central sensitization pain sensitivity among individuals with symptomatic knee. Arthritis Care Res (Hoboken). 2016;68(4):472-80.
10. Kellgren JH, Lawrence JS. Radiological assessment of osteo-arthrosis. Ann Rheum Dis. 1957;16(4):494-502.

11. Scott J, Huskisson EC. Graphic representation of pain. Pain. 1976;2:175-84.

12. Snaith RP. The hospital anxiety and depression scale. Health Qual Life Outcomes. 2003;1:29.

13. Lundeberg T, Lund I, Dahlin L, Borg E, Gustafsson C, Sandin L, et al. Reliability and responsiveness of three different pain assessments. J Rehabil Med. 2011;33:279-83.

14. Roos $\mathrm{PH}$, Laurèn $\mathrm{M}$, Adalberth $\mathrm{T}$, Roos EM, Jonsson $\mathrm{K}$, Lohmander LS. Knee osteoarthritis after meniscectomy. Arthritis Rheum. 1998;41:687-93.

15. Kinser AM, Sands WA, Stone MH. Reliability and validity of a pressure algometer. J Strength Cond Res. 2009;23(1):312-4.

16. Torstensen TA, Grooten W, Østerås H, Heijne A, Harms-Ringdahl K, Äng BO. How does exercise dose affect patients with long-term osteoarthritis of the knee? A study protocol of a randomized controlled trial in Sweden and Norway: the SWENOR Study. BMJ Open. 2018;8:e018471. https://doi.org/10. 1136/bmjopen-2017-018471.

17. Lorås $\mathrm{H}$, Østerås $\mathrm{B}$, Torstensen TA, Østerås $\mathrm{H}$. Medical exercise therapy for treating musculoskeletal pain: a narrative review of results from randomized controlled trials with a theoretical perspective. Physiother Res Int. 2015;20(3):182-90.

18. Teders SJ, Blanchard EB, Andrasik F, Jurish SE, Neff DF, Arena JG. Relaxation training for tension headache: comparative efficacy and cost-effectiveness of a minimal therapist contact versus a therapist-delivered procedure. Behav Ther. 1984;15:59-70.

19. Kashima K, Rahman OI, Sakoda S, Shiba R. Increased pain sensitivity of the upper extremities of TMD patients with myalgia to experimentallyevoked noxious stimulation: possibility of worsened endogenous opioid systems. Cranio. 1999;17:241-6.

20. Leffler AS, Hansson P, Kosek E. Somatosensory perception in a remote pain-free area and function of diffuse noxious inhibitory controls (DNIC) in patients suffering from long-term trapezius myalgia. Eur J Pain. 2002;6:149-59.

21. Kumazawa T. Primitivism and plasticity of painimplication of polymodal receptors. Neurosci Res. 1998;32:9-31. 
22. Iannetti GD, Mouraux A. From the neuromatrix to the pain matrix (and back). Exp Brain Res. 2010;205(1):1-12.

23. Gold MS, Gebhart GF. Nociceptor sensitization in pain pathogenesis. Nat Med. 2010;16(11):1248-57.

24. Fingleton $\mathrm{C}$, et al. Pain sensitization in people with knee osteoarthritis: a systematic review and metaanalysis. Osteoarthr Cartil. 2015;23(7):1043-56.

25. Thorstensson CA, et al. Natural course of knee osteoarthritis in middle-aged subjects with knee pain: 12-year follow-up using clinical and radiographic criteria. Ann Rheum Dis. 2009;68(12):1890-3.

26. Ambrose KR, Golightly YM. Physical exercise as non-pharmacological treatment of chronic pain: why and when. Best Pract Res Clin Rheumatol. 2015;29(1):120-30.

27. Naugle KM, Fillingim RB, Riley JL. A meta-analytic review of the hypoalgesic effects of exercise. J Pain. 2012;13(12):1139-50.

28. Bergmann N, Ballegaard S, Holmager P, et al. Pressure pain sensitivity: a new method of stress measurement in patients with ischemic heart disease. Scand J Clin Lab Investig. 2013;73(5):773-9.

29. Cunha GO, Pinto-Fiamengui L, Sampaio F, Conti P. Is aerobic exercise useful to manage chronic pain? Rev Dor São Paulo. 2016;17(1):61-4.
30. Reddy KS, Naidu MU, Rani PU, Rao TR. Human experimental pain models: a review of standardized methods in drug development. J Res Med Sci. 2012;17(6):587-95.

31. Chapman CR, Donaldson GW, Nakamura Y, Jacobson RC, Bradshaw DH, Gavrin J. A psychophysiological causal model of pain report validity. J Pain. 2002;3:143-55.

32. Vaegter HB, Handberg G, Graven-Nielsen T. Isometric exercises reduce temporal summation of pressure pain in humans. Eur J Pain. 2015;19:973-83.

33. Cooper MA, Kluding PM, Wright DE. Emerging relationships between exercise, sensory nerves, and neuropathic pain. Front Neurosci. 2016;10:372.

34. Fransen M, McConnell S, Harmer AR, Van der Esch M, Simic M, Bennell KL. Exercise for osteoarthritis of the knee: a Cochrane systematic review. $\mathrm{Br} \mathrm{J}$ Sports Med. 2015;49:1554-7.

35. McAlindon TE, Bannuru RR, Sullivan MC, Arden NK, Berenbaum F, Bierma-Zeinstra SM, Hawker GA, Henrotin Y, Hunter DJ, Kawaguchi H, et al. OARSI guidelines for the non-surgical management of knee osteoarthritis. Osteoarthr Cartil. 2014;22:363-88. 